\title{
The complete mitochondrial genome of Anoplocephala perfoliata, the first representative for the family Anoplocephalidae
}

\author{
Aijiang Guo ${ }^{1,2}$
}

\begin{abstract}
Background: Mitochondrial (mt) genome sequences are widely used to understand phylogenetic relationships among parasites. However, no complete $\mathrm{mt}$ genome sequence is available in the family Anoplocephalidae to date. This study sequenced and annotated the complete mt genome of Anoplocephala perfoliata (Anoplocephalidae), and investigated its phylogenetic relationships with other species from the families Hymenolepididae, Dipylidiidae and Taeniidae of the order Cyclophyllidea using the amino acid sequences of the 12 proteins in their mt genomes.

Methods: The complete mt genome of A. perfoliata was amplified by Long-range PCR, sequenced using primer walking and annotated by comparing with those of other cestodes. Its phylogenetic relationship with the species from the families Hymenolepididae, Dipylidiidae and Taeniidae was inferred using the 12 protein sequences based on Maximum likelihood and Bayesian methods.

Results: The complete circular mt genome sequence for A. perfoliata is 14,459 bp in size, and includes 12 protein-coding genes, 2 rRNA genes and 22 tRNA genes. The mt gene arrangement of $A$. perfoliata is identical to those of previously reported Hymenolepis diminuta (Hymenolepididae) and Dipylidium caninum (Dipylidiidae), but slightly different from those of other taeniids due to an order switch between tRNA(S2) and tRNA(L1). The phylogenetic analyses showed that the Dipylidiidae was more closely related to Anoplocephalidae and Hymenolepididae than to Taeniidae. The relationship among the four families obtained by Maximum likelihood and Bayesian inferences based on predicted amino acid sequences of protein-coding genes is consistent with that based on their mt gene arrangement similarities.

Conclusions: This study determined the first mt genome for the family Anoplocephalidae, providing rich sources for selecting useful molecular markers for ecological and phylogenetic studies. Analyses on mt genome sequences of the four families of cestodes provide novel insights into their phylogenetic relationships. Of couse, more taxon sampling is necessary for future phylogenetic studies of these cestodes using mt genome sequences.
\end{abstract}

Keywords: Anoplocephala perfoliata, Mitochondrial genome, Phylogenetic analysis

\section{Background}

The Cyclophyllidea order with 15 families is the biggest taxonomic group in Cestoda. The phylogenies of cyclophyllidean tapeworms have been widely studied based on morphological characters $[1,2]$ which are often convergent and

\footnotetext{
Correspondence: guoaijiang@caas.cn

${ }^{1}$ State Key Laboratory of Veterinary Etiological Biology, Key Laboratory of Veterinary Parasitology of Gansu Province, Lanzhou Veterinary Research Institute, Chinese Academy of Agricultural Sciences, Lanzhou 730046Gansu Province, People's Republic of China

${ }^{2}$ Jiangsu Co-innovation Center for Prevention and Control of Important Animal Infectious Diseases and Zoonoses, Yangzhou 225009jiangsu Province, People's Republic of China
}

() Biomed Central

(c) 2015 Guo. Open Access This article is distributed under the terms of the Creative Commons Attribution 4.0 International License (http://creativecommons.org/licenses/by/4.0/), which permits unrestricted use, distribution, and reproduction in any medium, provided you give appropriate credit to the original author(s) and the source, provide a link to the Creative Commons license, and indicate if changes were made. The Creative Commons Public Domain Dedication waiver (http:// creativecommons.org/publicdomain/zero/1.0/) applies to the data made available in this article, unless otherwise stated. similarities are observed between the intermicapsiferine anoplocephalids and the davaineids with fibrous polyovular egg capsules. The Anoplocephalidae is diagnosed in part by the absence of a rostellum when compared with the davaineids. However, the rostellum has been secondarily lost in numerous species or genera within the Davaineidae [1].

Molecular tools, using specific genes and genomic regions (12S rDNA, 28S rDNA, cox1, etc.) [3, 5-10], have been used to study phylogenetic relationships among groups of parasitic flatworms. However, some specific genes and genomic regions such as rDNA genes, may 
not completely provide the useful phylogenetic information at the generic level $[6,11]$, or may not be the most suitable marker for all taxa $[12,13]$. Nevertheless, complete mitochondrial DNA sequences have become effective analytical tools in ecology, evolutionary biology and population genetics [14-18]. The complete mt genomes provide not only individual and combined mtDNA molecular markers for within- and between-species studies but also the information of gene order changes [19]. Platyhelminthe mt genomes comprise 12 protein-coding genes, 2 ribosomal and 22 transfer RNA genes [15], but lack the atp 8 gene. In general, the mitochondrial gene order is rather stable and gene rearrangements are rare evolutionary events. When any common change is discovered within a group, it is considered to be a phylogenetic signal $[15,20,21]$. Although $\mathrm{mt}$ genome data provide useful molecular markers for population genetics and phylogenetic studies, there are some families in the cestode order Cyclophyllidea for which mtDNAs are still unavailable to date. Such, few studies on the phylogeny of cyclophyllidean cestodes are conducted at the family level based on complete mitochondrial DNA sequences [11, 14].

Complete mitochondrial genome is unavailable for the Anoplocephalidae family as yet and its systemic relationships with other cestodes in the same order of Cyclophyllidea are currently poorly understood. Several species that belong to the family Anoplocephalidae can cause tapeworm infections in horses and donkeys [22]. Among them, Anoplocephala perfoliata is a notable one with a worldwide distribution [23]. In the present study, the complete mitochondrial genome of A. perfoliata (Anoplocephalidae) has been sequenced and annotated, which is the first representative of the family Anoplocephalidae. The A. perfoliata mt DNA sequence was used along with those of nine published $\mathrm{mt}$ genomes of species of cestode families in the Cyclophyllidea, including Hymenolepis diminuta (the only complete mt genome available in Hymenolepididae), Dipylidium caninum (the only complete mt genome available in Dipylidiidae), Versteria mustelae, Echinococcus granulosus, Echinococcus multilocularis, Taenia solium, Taenia asiatica, Hydatigera krepkogorski and Hydatigera parva (seven representative $\mathrm{mt}$ genome sequences from the four genera of Taeniidae) to explore family-level phylogenetic relationships of the four families Anoplocephalidae, Taeniidae, Dipylidiidae and Hymenolepididae.

\section{Methods}

\section{Sampling and DNA extraction}

Single $A$. perfoliata adult was collected from the digestive tract of a donkey slaughtered at a commercial abattoir in China for DNA extraction and sequencing. The tapeworm was washed with $0.75 \% \mathrm{NaCl}$. The genomic DNA was extracted using Multisource Genomic
Miniprep DNA extraction kit (AXYGEN, USA) following the manufacturer's instructions.

\section{Amplification of mtDNA fragments}

The complete mitochondrial genomic DNA was amplified by PCR in three overlapping fragments (nad1-rrnS, rrnS-nad5, nad5-nad1) using three pairs of primers (Additional file 1: Table S4), which were designed according to the published primers [14] used for amplifying short fragments of mtDNAs for all Taenia species. PCR reactions were carried out in a $50 \mu \mathrm{l}$ reaction mixture consisting of $25 \mu \mathrm{l} 2 \times$ buffer (Takara Biotechnology Co. Ltd., Dalian, China), $22.5 \mu$ l sterile deionized water, $0.5 \mu \mathrm{l}$ each primer $(50 \mathrm{pmol} / \mu \mathrm{l})$, and $1.5 \mu \mathrm{l}$ DNA template $(40 \mathrm{ng} / \mu \mathrm{l})$ in the following conditions: $94{ }^{\circ} \mathrm{C}$ for $5 \mathrm{~min}$, then $94{ }^{\circ} \mathrm{C}$ for $30 \mathrm{~s}, 50{ }^{\circ} \mathrm{C}$ for $30 \mathrm{~s}, 68^{\circ} \mathrm{C}$ for $8 \mathrm{~min}$ for 30 cycles, followed by $68^{\circ} \mathrm{C}$ for $10 \mathrm{~min}$. PCR products were purified and underwent sequencing. Each amplicon was sequenced in both directions by primer walking [24] with ABI PRISM 3730 (Applied Biosystems Inc.) at Takara Biotechnology Co. and Shanghai Sangon Co.

\section{Sequence analyses}

The complete mt genomic sequence of $A$. perfoliata was assembled using CAP3 Sequence Assembly Program [25] (http://doua.prabi.fr/software/cap3) and annotated by sequence comparison with those of other cestodes $[14,16]$ available in the GenBank database. Proteincoding genes were identified using BLAST searches and ORF Finder software at NCBI with the genetic code set for Flatworm (Translation table 9). Transfer RNA (tRNA) genes were annotated using tRNAscan-SE, combining with recognizing anticodon sequences and potential secondary structures by visual inspection. The ribosomal RNA genes (rRNA) were identified by sequence comparisons with the published cestode mt rRNA genes. Palindromes and inverted sequences in the non-coding regions were determined using Einverted and Palindrome in EMBOSS software [26]. Secondary structures were predicted using Mfold software [27]. Tandem repetitive elements were detected with Tandem Repeats Finder program [28]. Nucleotide composition (\%) of each gene, non-coding region and the complete $\mathrm{mt}$ genome was calculated using DNASTAR's Lasergene sequence analysis software [29].

\section{Phylogenetic analyses}

A total of $16 \mathrm{mt}$ genome sequences were used for analysis, including seven representative $\mathrm{mt}$ genome sequences from the four genera of Taeniidae, and two cyclophyllidean cestode mt genomes available in GenBank (Hymenolepis diminuta (Hymenolepididae), and Dipylidium caninum (Dipylidiidae)), the $\mathrm{mt}$ genome sequence for $A$. perfoliata identified in this study, five $\mathrm{mt}$ genomes from Pseudophyiidea (Spirometra erinaceieuropaei, Diphyllobothrium 
nihonkaiense, Kiphyllobothrium latum, Diplogonoporus balaenopterae and Diplogonoporus grandis), and $\mathrm{mt}$ sequences from the trematode Schistosoma japonicum (outgroup) (Additional file 1: Table S1). Phylogenetic analyses were carried out using amino acid sequences, which were predicted from the nucleotide sequences of each proteincoding gene using the flatworm mt genetic code set (Translation table 9). The amino acid sequences for each proteincoding gene of 16 species were aligned individually using MAFFT 7.122 [30]. Poorly aligned positions were removed by Gblocks server (http://molevol.cmima.csic.es/castresana/ Gblocks_server.html) [31] using the option for a less stringent selection. A single alignment for phylogenetic analysis was created by concatenating all amino acid alignments of the 12 protein-coding genes. The optimal model of protein evolution was selected by ProtTest [32]. The phylogenetic trees were constructed using Bayesian analysis and Maximum-likelihood (ML). Bayesian analysis was performed by MrBayes 3.2 [33] with mtZoa model (rates = gamma, ngammacat $=5$ ), as suggested by Rota-Stabelli et al. [34]. Two chains (temp $=0.2$ ) were run for $5,000,000$ generations and sampled every 1,000 generations. The first $25 \%$ of the trees were treated as burn-in. ML analysis were conducted with PhyML 3.0 [35] using the MtArt + I + G model, and 100 bootstrap replicates were selected to calculated bootstrap support for ML trees.

\section{Results and discussion}

The complete $\mathrm{mt}$ genome organization of $A$. perfoliata

The complete $\mathrm{mt}$ genome of $A$. perfoliata (GenBank: KR054960) is a circular, double-stranded DNA molecule with $14,459 \mathrm{bp}$ in size (Fig. 1), which is slightly larger than those of other cestodes (13,482 bp-14,296 bp) $[14,36]$ (Additional file 1: Table S1). High A + T content (70.99 \%) and low $\mathrm{G}+\mathrm{C}$ content (29.01\%) were observed in the $\mathrm{mt}$ genome sequence of $A$. perfoliata (Additional file 1: Table S2). The overall $\mathrm{A}+\mathrm{T}$ content is very similar to those of other cestode species reported so far (H. diminuta [71.04 \%], D. caninum [72.89\%], T. solium [72.1\%], T. asiatica [71.4 \%], E. multilocularis [69.04\%] and E. granulosus [67.02\%]). The 12 protein-coding genes (cox1-3, atp6, nad1-6, nad4L and cytb), 22 tRNA genes and two rRNA genes $(r r n S$ and $r r n L)$ were identified in A. perfoliata $\mathrm{mt}$ genome by comparing their sequence identities and/or secondary structures with those of other flatworms. The atp8 gene that appears in most animal mtDNAs is missing in this A. perfoliata isolate, as in other known flatworm $\mathrm{mt}$ genomes. All the genes are encoded by one strand in the same direction (Fig. 1). A list of gene arrangement, gene length and intergenic spacer regions of mt genome for $A$. perfoliata are shown in Table 1 . It is noteworthy that the gene order of $A$. perfoliata $\mathrm{mt}$ genome is identical to those of $H$. diminuta and D. caninum, but slightly different from

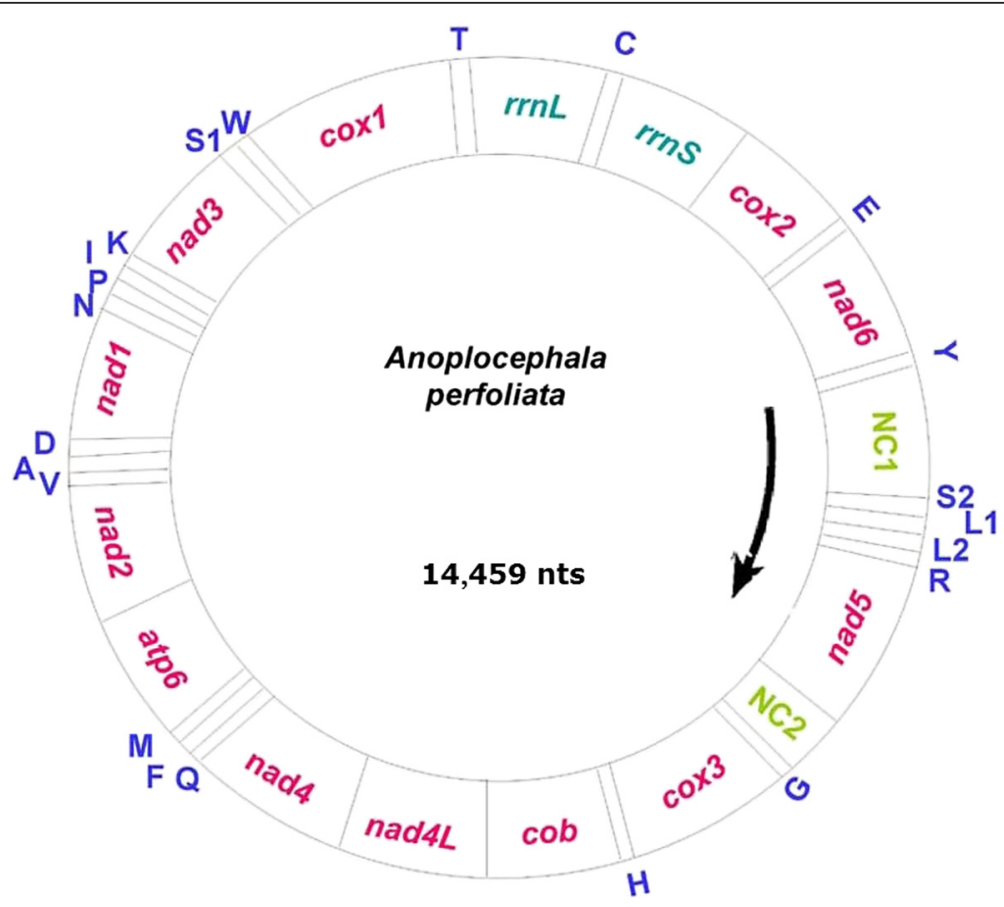

Fig. 1 Structure of the mitochondrial genome of Anoplocephala perfoliata (also see Table 1). Genome organization of the complete mitochondrial genome of A. perfoliata is a circular DNA molecule, which contains 36 genes. All genes are coded by the same DNA strand. Arrow indicates transcriptional orientation. NC refers to non-coding region. 22 tRNA genes are designated with one letter amino acid codes. Gene scaling is approximate 
Table 1 List of annotated mitochondrial genome of Anoplocephala perfoliata

\begin{tabular}{|c|c|c|c|c|c|c|}
\hline Genes & Positions & Lengths (bp) & Initiation and termination codons & Anticodons & Number of aa & Intergenic nucleotides $(\mathrm{bp})^{\mathrm{a}}$ \\
\hline $\operatorname{cox} 1$ & $1-1593$ & 1593 & ATG/TAA & - & 530 & 5 \\
\hline tRNA-Thr (T) & $1596-1659$ & 64 & - & TGT & - & 2 \\
\hline$r r n L$ & $1660-2640$ & 981 & - & - & - & 0 \\
\hline tRNA-Cys (C) & $2641-2706$ & 66 & - & GCA & - & 0 \\
\hline$r r n s$ & $2707-3430$ & 724 & - & - & - & 0 \\
\hline $\operatorname{cox} 2$ & $3431-4006$ & 576 & ATG/TAG & - & 191 & 0 \\
\hline tRNA-Glu (E) & $4008-4071$ & 64 & - & $\pi \mathrm{TC}$ & - & 1 \\
\hline nad6 & $4076-4534$ & 459 & ATG/TAG & - & 152 & 4 \\
\hline tRNA-Tyr (Y) & $4541-4606$ & 66 & - & GTA & - & 6 \\
\hline Non-coding region (NC1) & $4607-5481$ & 875 & - & - & - & 0 \\
\hline tRNA-SerUCN (S2) & $5482-5554$ & 73 & - & TGA & - & 0 \\
\hline tRNA-LeuCUN (L1) & $5582-5642$ & 61 & - & TAG & - & 27 \\
\hline tRNA-LeuUUR (L2) & $5654-5717$ & 64 & - & TAA & - & 11 \\
\hline tRNA-Arg (R) & $5727-5783$ & 57 & - & ACG & - & 9 \\
\hline nad5 & $5785-7365$ & 1581 & ATG/TAG & - & 526 & 1 \\
\hline Non-coding region (NC2) & $7366-7644$ & 279 & - & - & - & 0 \\
\hline tRNA-Gly (G) & $7642-7704$ & 63 & - & TCC & - & -3 \\
\hline $\operatorname{cox} 3$ & $7708-8351$ & 644 & ATG/TA & - & 214 & 3 \\
\hline tRNA-His $(\mathrm{H})$ & $8352-8418$ & 67 & - & GTG & - & 0 \\
\hline cytb & $8422-9522$ & 1101 & GTG/TAG & - & 366 & 3 \\
\hline nad4L & $9525-9785$ & 261 & ATG/TAG & - & 86 & 2 \\
\hline nad4 & 9752-10999 & 1248 & ATG/TAA & - & 415 & -34 \\
\hline tRNA-Gln (Q) & $11001-11063$ & 63 & - & TTG & - & 1 \\
\hline tRNA-Phe (F) & 11062-11123 & 62 & - & GAA & - & -2 \\
\hline tRNA-Met (M) & $11120-11184$ & 65 & - & CAT & - & -4 \\
\hline atp6 & 11188-11703 & 516 & ATG/TAG & - & 171 & 3 \\
\hline nad2 & $11718-12593$ & 876 & ATG/TAG & - & 291 & 14 \\
\hline tRNA-Val (V) & $12598-12660$ & 63 & - & TAC & - & 4 \\
\hline tRNA-Ala (A) & $12660-12724$ & 65 & - & TGC & - & -1 \\
\hline tRNA-Asp (D) & $12726-12789$ & 64 & - & GTC & - & 1 \\
\hline nad1 & 12793-13683 & 891 & ATG/TAA & - & 296 & 3 \\
\hline tRNA-Asn (N) & 13710-13778 & 69 & - & $\mathrm{GTT}$ & - & 26 \\
\hline tRNA-Pro (P) & 13784-13849 & 66 & - & TGG & - & 5 \\
\hline tRNA-lle (I) & 13850-13912 & 63 & - & GAT & - & 0 \\
\hline tRNA-Lys (K) & 13917-13978 & 62 & - & $\mathrm{CTT}$ & - & 4 \\
\hline nad3 & 13983-14330 & 348 & ATG/TAA & - & 115 & 4 \\
\hline tRNA-SerAGN (S1) & $14331-14390$ & 60 & - & $\mathrm{GCT}$ & - & 0 \\
\hline tRNA-Trp (W) & $14393-14454$ & 62 & - & TCA & - & 2 \\
\hline
\end{tabular}

Notes: ${ }^{\text {a }}$ shows the length of overlap (-) or intergenic gap (+) between two adjacent genes

those of other taeniids due to an order switch between tRNA(S2) and tRNA(L1).

\section{Protein-coding genes}

For the 12 protein-coding genes identified in A perfoliata, the $c y t b(1,101 \mathrm{bp})$ is relatively large in size, whereas cox 1
(1,593 bp) is among the smallest and the remaining ten genes are comparable with the normative sizes, as with the other cestodes $\mathrm{mt}$ genomes thus far characterized (Additional file 1: Table S3). Initiation and termination codons inferred from each protein-coding gene are shown in Table 1. Of 12 protein-coding genes, eleven genes were 
inferred to initiate with the ATG codon except that $c y t b$ is probably initiated with GTG. Seven were inferred to use TAG as stop codons (cox2, nad6, nad5, cytb, nad4L, atp6 and $n a d 2$ ), four were inferred to terminate with TAA ( cox1, nad4, nad1 and nad3) and one gene (cox3) was terminated with an incomplete stop codon (TA). The truncated incomplete stop codon has also been found in cox3 gene for $D$. caninum. The initiation and complete or incomplete termination codons for $A$. perfoliata are as normal for flatworm mitochondrial protein-coding genes (Additional file 1: Table S3). Gene overlaps were found between tRNA $(\mathrm{F})$ and $\operatorname{tRNA}(\mathrm{Q})$, between $\operatorname{tRNA}(\mathrm{Q})$ and tRNA(M), and between tRNA(V) and tRNA(A). The nad4 gene is inferred to overlap with the upstream nad4L gene by 34 nucleotides, which is common for these two genes among metazoan mtDNAs [16].

\section{Ribosomal RNA and transfer RNA genes}

The two ribosomal RNA genes, $r r n S$ and $r r n L$, were predicted from the mt genome of A. perfoliata. rrnS is located between $\operatorname{tRNA}(\mathrm{C})$ and $c o x 2, r r n L$ is found between tRNA(T) and tRNA $(\mathrm{C})$, the same position as reported in other cestodes [14, 16]. The length of $r r n L(992 \mathrm{bp})$ is relatively larger and $r r n S(724 \mathrm{bp})$ is normal, compared to those found in some other cestode species (Additional file 1: Table S3). The A + T contents of $r r n S$ and $r r n L$ are $69.34 \%$ and $70.67 \%$, respectively.

The 22 tRNA genes were identified in the mtDNA of A. perfoliata. The sizes of the tRNA genes are $57-73 \mathrm{bp}$ (Table 1). Predicted secondary structures of 18 tRNAs have the standard cloverleaf structure in A. perfoliata, but that of four tRNA genes $(\operatorname{tRNA}(\mathrm{C})$, tRNA(R) and two tRNA(S)) lack the DHU arm (Fig. 2), as reported in some Taenia species mtDNAs [14]. However, in the
tRNAs of the $H$. diminuta mt genome, only three tRNAs (the tRNA(R) and the two tRNA(S) genes) lack the DHU arm. Each of the four tRNAs has the potential for an extra base-pairing, probably allowing a longer anticodon stem in A. perfoliata (Fig. 2). Similar tRNA structures with a longer anticodon stem are also found in Taenia species [14] and $H$. diminuta mtDNA [16]. Additionally, it is noteworthy that an additional base-pairing for a longer acceptor stem may also be possible for the tRNA(C) in A. perfoliata (Fig. 2), yet no similar structure has been reported in other cestode mtDNAs.

\section{Non-coding sequences}

The mt genome of $A$. perfoliata contained two major noncoding regions (NC1 or NC2) (Table 1). NC1 (875 bp) is located between tRNA(Y) and tRNA(S2). NC2 (279 bp) is found between $\operatorname{nad} 5$ and $\operatorname{tRNA}(\mathrm{G})$. These are at the same relative positions as in the $\mathrm{mt}$ genome of $H$. diminuta [16], but $\mathrm{NC} 1$ is larger than $\mathrm{NC} 2$ in the A. perfoliata mt genome, whereas $\mathrm{NC} 1$ is smaller than $\mathrm{NC} 2$ in the H. diminuta mt genome (Additional file 1: Table S3). These differences in length between the two non-coding regions are less pronounced in cestodes and may not be used as phylogenetic signals [15]. For the other reported taeniid mtDNAs [14], however, the two non-coding regions are placed between tRNA(Y) and tRNA(L1), and between nad5 and tRNA(G), respectively.

In the $A$. perfoliata $\mathrm{mt}$ genome, $\mathrm{NC1}$ is mainly composed of four repeat elements of $169 \mathrm{bp}$, plus a part of the repeat element of $85 \mathrm{bp}$. Only two bases vary among these four repeat elements. The presence of long repeats is a common feature of non-coding regions for cestodes [16]. A number of potential secondary structures in the $\mathrm{NC1}$ of A. perfoliata were also predicted. For example, a 
stable potential secondary structure with two arms and two loops could be folded in each of these repeat regions (Fig. 3a). This secondary structure contains 11 and five canonical base pairs for the stems, with $3 \mathrm{bp}$ and $5 \mathrm{bp}$ loops, respectively (Fig. 3a). For NC1, three two-stem structures are feasible, but a large potential secondary structure presented as a pentamer is also possible with 24 canonical base pairs plus long loops (Fig. 3a). The large potential secondary structures predicted in triplex have been observed for non-coding regions between nad5 and tRNA(G) or between tRNA(Y) and tRNA(L1) in other cestode mtDNAs [16]. Competitive and mutually exclusive hairpins could be present in this case, as reported in other mtDNAs [16, 37]. Besides the aforementioned major repeat regions, there were two sets of short inverted sequences at the NC1 terminals (TAATAC TATTA and ATAAATTTAAAAATTTAT), and each of them could be folded into a hairpin structure.

The second non-coding region (NC2) of the mt genome of $A$. perfoliata comprises six identical repeat elements of a $32 \mathrm{bp}$ nucleotide plus one shuffling repeat element (Fig. 3b). Except for these repeat sequences, the other non-coding sequence with $52 \mathrm{bp}$ in $\mathrm{NC} 2$ can form a stem-loop structure with 15 standard base pairs and a loop of 16 bp nucleotides (Fig. 3b). This structure, including identical repeats and a stem-loop in its non-coding region, is only observed in the published mt genome sequences of $H$. diminuta and Meloidogyne javanica, which have been predicted for 13 repeat elements with a stem-loop and 11 repeat elements with a stem-loop, respectively $[16,38]$.

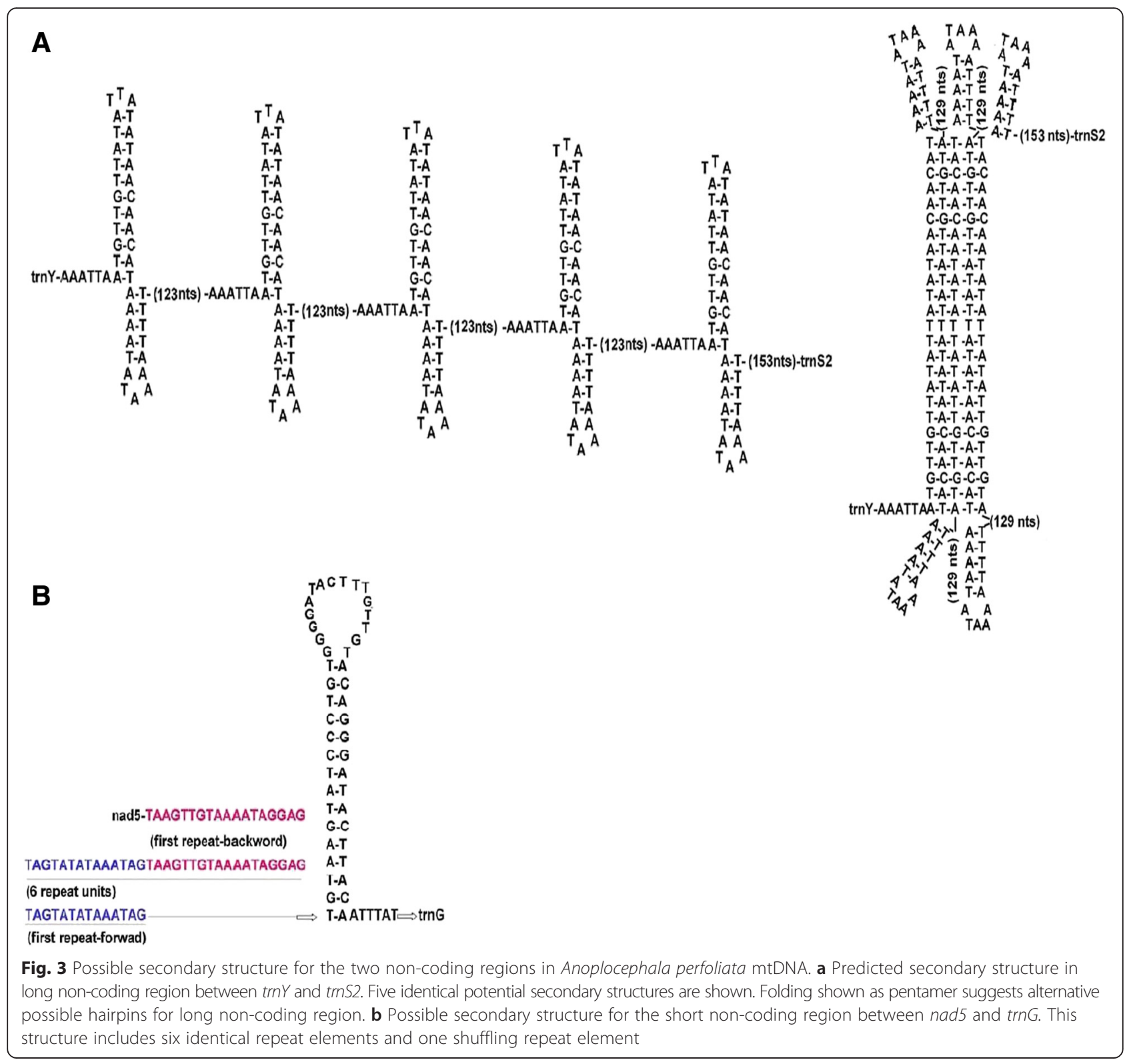


The lengths of non-coding regions in other cestode mt genomes are shown in Additional file 1: Table S3. Similar and stable stem-loop structures can be also found in the non-coding regions of taeniid mtDNAs [14], though the functions of these non-coding regions remain unclear. In mammals, however, similar stable hairpin structures have been shown to initiate replication and transcription $[39,40]$. Whether these non-coding regions of cestode mtDNAs serve a similar function remains to be investigated. Other small intergenic regions were discovered between tRNA(S2) and tRNA(L1) (30 bp) and between nad 1 and tRNA(N) (26 bp). Whether any of these noncoding regions serve any function awaits investigation.

\section{Phylogenetic analyses}

In order to evaluate the phylogenetic relationship among the families of the order Cyclophyllidea based on $\mathrm{mt}$ genome data, phylogeny was reconstructed using inferred amino acid sequences of the 12 protein-coding genes from 16 representatives including ten species from the order Cyclophyllidea, five species from the order Pseudophyllidea and one trematode outgroup. An identical phylogenetic tree topology was obtained through the Maximumlikelihood (ML) and Bayesian analyses (Fig. 4). The tree inferred using the ML method was the same as that of the Bayesian analysis and was omitted. The trees showed that Pseudophyllidea and Cyclophyllidea individuals formed a monophyletic group which is consistent with previous molecular analyses $[11,41,42]$. Within the Taeniidae group, four valid genera were robustly supported with high nodal support. This result is in agreement with the study by
Nakao et al. [11], but different from those of other studies suggesting that the cestode family Taeniidae has only two valid genera [11]. The tree revealed sister-group relationship between the Anoplocephalidae and the Hymenolepididae, which agrees with what has been reported using the $18 \mathrm{~S}$ gene based on distance-based NeighbourJoining method, but is not consistent with the parsimonybased phylogeny [6]. This inferred relationship between the Anoplocephalidae and the Hymenolepididae is also consistent with some earlier morphological and molecular studies (12S rDNA and cox1) suggesting both families as very close parents [3, 5]. Furthermore, the trees indicated that the Dipylidiidae was phylogenetically closer to the Hymenolepididae and the Anoplocephalidae than to the Taeniidae. The evolutionary relationship between these four families revealed in the present study is incongruent with the hypothesis based on the phylogenetic tree derived from 12S rDNA [3] which suggests that the Dipylidiidae is more closely related to the Taeniidae than to either the Hymenolepididae or the Anoplocephalidae, and also disagrees with Hoberg's proposal [1] that the Hymenolepididae is closer to the Dipylidiidae and the Taeniidae than to the Anoplocephalidae based on comparative morphology analysis.

The present study is the first one to investigate the relationship among these four families using concatenated $\mathrm{mt}$ amino acid sequences of 12 protein-coding genes. The relationship among these four families obtained here shows that the Dipylidiidae is more closely related to the Hymenolepididae and the Anoplocephalidae than to the Taeniidae. This can also be supported by the analysis based on similarities of their mt gene arrangement, where the gene
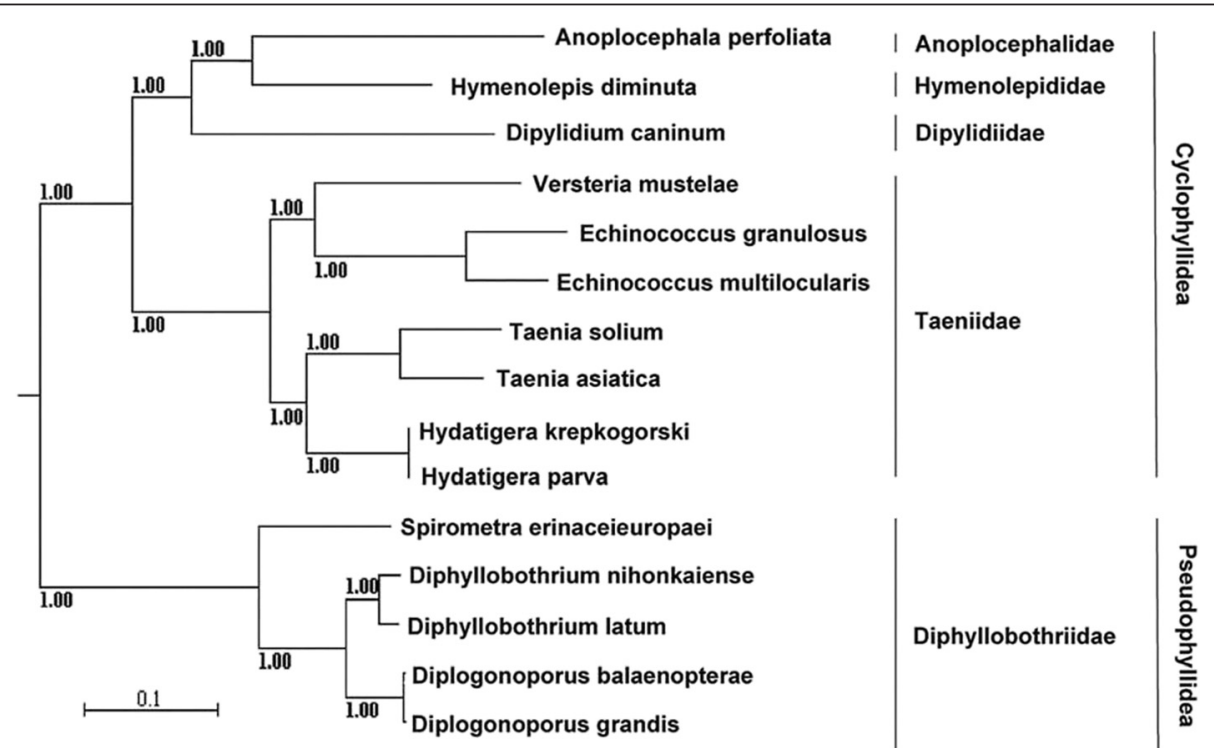

Fig. 4 Phylogenetic relationships between Anoplocephalidae, Hymenolepididae, Dipylidiidae and Taeniidae. Phylogenetic tree inferred by the Bayesian inference analysis from deduced amino acids of 12 protein-coding genes is shown. Scale bar indicates number of substitutions per site. Posterior probability values are shown 
arrangement of $A$. perfoliata (Anoplocephalidae) mt genome is identical to those of the species of $H$. diminuta (Hymenolepididae) and D. caninum (Dipylidiidae), but different from those of other taeniids. The identical $\mathrm{mt}$ gene arrangement among the species of the families Anoplocephalidae, Hymenolepididae and Dipylidiidae supported the result from the Bayesian phylogeny based on concatenated amino acids that these three families might have a closer relationship. Additionally, sequence organization for one of the two non-coding regions in $A$. perfoliata is very similar to that of $H$. diminuta, where the front part of the non-coding region comprises 6 identical repeat elements and the remaining non-coding region sequence can form a stem-loop structure in A. perfoliata (Fig. 3b), and 13 repeat elements with a stem-loop structure in $H$. diminuta $[16,38]$. Whether the similar feature of the non-coding region between $A$. perfoliata and $H$. diminuta can be used as phylogenetic marker to support the closer relationship between the Anoplocephalidae and the Hymenolepididae is not known yet, a wider sampling will be required to determine. In comparison with other cyclophyllidean tapeworms described earlier $[14,16,18,43]$, mitochondrial genome organization and structure of $A$. perfoliata are more similar with them, supporting the result from the Bayesian phylogeny that the family Anoplocephalidae belongs to the order Cyclophyllidea. Considering the limited $\mathrm{mt}$ genome data available for these cestode parasites, additional taxon sampling of the families in the order Cyclophyllidea including the Anoplocephalidae, the Hymenolepididae and the Dipylidiidae is required to fully assess of the inferred phylogenetic relationship.

\section{Conclusions}

In conclusion, this study details the complete sequencing and annotating of the mt genome of A. perfoliata. This represents the first complete mtDNA sequence from the family Anoplocephalidae. The complete mtDNA genome contains 36 genes, including 12 protein-coding genes, two ribosomal RNA genes and 22 transfer RNA genes. Phylogenetic analysis inferred from amino acid sequences of 12 protein-coding genes from ten representatives belonging to four families (Hymenolepididae, Dipylidiidae, Taeniidae and Anoplocephalidae) showed that the Dipylidiidae is more closely related to the Hymenolepididae and the Anoplocephalidae than to the Taeniidae. This can also be inferred from the similarities of their mt gene arrangement. This finding provides novel insights into the phylogenetic hypothesis of the four major families of the order Cyclophyllidea. The complete $A$. perfoliata mt genome determined in the present study will provide novel $\mathrm{mt}$ genetic markers for studies of the equine tapeworm in epidemiology, molecular identification and diagnosis, and also help to reassess the systematic relationships of cestodes in the order Cyclophyllidea.

\section{Additional file}

Additional file 1:Table S1. Cestode species used for comparative analysis with Anoplocephala. perfoliata. Table S2. A + T content (\%) of the protein-coding, tRNA, rRNA genes and non-coding regions of mitochondrial genome of Anoplocephala perfoliata. Table S3. Properties of mtDNA protein-coding genes, rRNA genes and non-coding regions of Anoplocephala perfoliata and other cestode species. Table S4. Primers used to amplify and sequence mitochondrial genome from Anoplocephala perfoliata. (DOC 209 kb)

\section{Competing interests}

The author declares that she has no competing interests.

\section{Acknowledgements}

This study was supported by the "National Key Basic Research Program (973 Program) of China" (Grant No. 2015CB150300). The author would like to express her appreciation to Dr. Shuai Wang, Ms Blinda Brice, and Dr. Guo-Hua Liu for critical reading and advice on this manuscript.

Received: 8 August 2015 Accepted: 15 October 2015

Published online: 21 October 2015

\section{References}

1. Hoberg EP, Jones A, Bray RA. Phylogenetic analysis among the families of the Cyclophyllidea (Eucestoda) based on comparative morphology, with new hypotheses for co-evolution in vertebrates. Syst Parasitol. 1999;42(1):51-73.

2. Hoberg EP, Gardner SL, Campbell RA. Systematics of the Eucestoda: advances toward a new phylogenetic paradigm, and observations on the early diversification of tapeworms and vertebrates. Syst Parasitol. 1999;42(1):1-12.

3. von Nickisch-Rosenegk M, Lucius R, Loos-Frank B. Contributions to the phylogeny of the Cyclophyllidea (Cestoda) inferred from mitochondrial $12 \mathrm{~S}$ rDNA. J Mol Evol. 1999;48(5):586-96.

4. Mariaux J. Cestode systematics: any progress? Int J Parasitol. 1996;26(3):231-43.

5. Haukisalmi V, Hardman LM, Hardman M, Laakkonen J, Niemimaa J, Henttonen H. Morphological and molecular characterisation of Paranoplocephala buryatiensis n. sp. and P. Iongivaginata Chechulin \& Gulyaev, 1998 (Cestoda: Anoplocephalidae) in voles of the genus Clethrionomys. Syst Parasitol. 2007;66(1):55-71.

6. Taleb-Hossenkhan N, Bhagwant S. Molecular characterization of the parasitic tapeworm Bertiella studeri from the island of Mauritius. Parasitol Res. 2012;110(2):759-68.

7. Mariaux J. A molecular phylogeny of the Eucestoda. J Parasitol. 1998:84(1):114-24.

8. Olson PD, Littlewood DT, Bray RA, Mariaux J. Interrelationships and evolution of the tapeworms (Platyhelminthes: Cestoda). Mol Phylogenet Evol. 2001;19(3):443-67.

9. Olson PD, Tkach W. Advances and trends in the molecular systematics of the parasitic Platyhelminthes. Adv Parasitol. 2005;60:165-243.

10. Lee SU, Chun HC, Huh S. Molecular phylogeny of parasitic Platyhelminthes based on sequences of partial $28 \mathrm{~S}$ rDNA D1 and mitochondrial cytochrome c oxidase subunit I. Korean J Parasitol. 2007;45(3):181-9.

11. Nakao M, Lavikainen A, Iwaki T, Haukisalmi V, Konyaev S, Oku Y, et al. Molecular phylogeny of the genus Taenia (Cestoda: Taeniidae): proposals for the resurrection of Hydatigera Lamarck, 1816 and the creation of a new genus Versteria. Int J Parasitol. 2013;43(6):427-37.

12. Will KW, Mishler BD, Wheeler QD. The perils of DNA barcoding and the need for integrative taxonomy. Syst Biol. 2005;54(5):844-51.

13. Zarowiecki MZ, Huyse T, Littlewood DT. Making the most of mitochondrial genomes-markers for phylogeny, molecular ecology and barcodes in Schistosoma (Platyhelminthes: Digenea). Int J Parasitol. 2007;37(12):1401-18.

14. Jia WZ, Yan HB, Guo AJ, Zhu XQ, Wang YC, Shi WG, et al. Complete mitochondrial genomes of Taenia multiceps, T. hydatigena and T. pisiformis: additional molecular markers for a tapeworm genus of human and animal health significance. BMC Genomics. 2010;11:447.

15. Littlewood DT, Lockyer AE, Webster BL, Johnston DA, Le TH. The complete mitochondrial genomes of Schistosoma haematobium and Schistosoma 
spindale and the evolutionary history of mitochondrial genome changes among parasitic flatworms. Mol Phylogenet Evol. 2006;39(2):452-67.

16. von Nickisch-Rosenegk M, Brown WM, Boore JL. Complete sequence of the mitochondrial genome of the tapeworm Hymenolepis diminuta: gene arrangements indicate that Platyhelminths are Eutrochozoans. Mol Biol Evol. 2001;18(5):721-30.

17. Jeon HK, Lee KH, Kim KH, Hwang UW, Eom KS. Complete sequence and structure of the mitochondrial genome of the human tapeworm, Taenia asiatica (Platyhelminthes; Cestoda). Parasitology. 2005;130(Pt 6):717-26.

18. Jeon HK, Kim KH, Eom KS. Complete sequence of the mitochondrial genome of Taenia saginata: comparison with T. solium and T. asiatica. Parasitol Int. 2007;56(3):243-6.

19. Le TH, Blair D, McManus DP. Mitochondrial genomes of parasitic flatworms. Trends Parasitol. 2002;18(5):206-13.

20. Boore JL, Brown WM. Big trees from little genomes: mitochondrial gene order as a phylogenetic tool. Curr Opin Genet Dev. 1998;8(6):668-74

21. Rokas A, Holland PW. Rare genomic changes as a tool for phylogenetics. Trends Ecol Evol. 2000;15(11):454-9.

22. Bohorquez GA, Luzon M, Martin-Hernandez R, Meana A. New multiplex PCR method for the simultaneous diagnosis of the three known species of equine tapeworm. Vet Parasitol. 2015;207(1-2):56-63.

23. Bohorquez A, Meana A, Pato NF, Luzon M. Coprologically diagnosing Anoplocephala perfoliata in the presence of A. magna. Vet Parasitol. 2014;204(3-4):396-401.

24. Hu M, Jex AR, Campbell BE, Gasser RB. Long PCR amplification of the entire mitochondrial genome from individual helminths for direct sequencing. Nat Protoc. 2007;2(10):2339-44.

25. Huang X, Madan A. CAP3: A DNA sequence assembly program. Genome Res. 1999;9(9):868-77.

26. Rice P, Longden I, Bleasby A. EMBOSS: the European Molecular Biology Open Software Suite. Trends Genet. 2000;16(6):276-7.

27. Zuker M. Mfold web server for nucleic acid folding and hybridization prediction. Nucleic Acids Res. 2003;31(13):3406-15.

28. Benson G. Tandem repeats finder: a program to analyze DNA sequences. Nucleic Acids Res. 1999;27(2):573-80.

29. Burland TG. DNASTAR's Lasergene sequence analysis software. Methods Mol Biol. 2000;132:71-91.

30. Katoh K, Standley DM. MAFFT multiple sequence alignment software version 7: improvements in performance and usability. Mol Biol Evol. 2013;30(4):772-80

31. Talavera G, Castresana J. Improvement of phylogenies after removing divergent and ambiguously aligned blocks from protein sequence alignments. Syst Biol. 2007;56(4):564-77.

32. Darriba D, Taboada GL, Doallo R, Posada D. ProtTest 3: fast selection of best-fit models of protein evolution. Bioinformatics. 2011;27(8):1164-5.

33. Ronquist F, Teslenko M, van der Mark P, Ayres DL, Darling A, Hohna S, et al. MrBayes 3.2: efficient Bayesian phylogenetic inference and model choice across a large model space. Syst Biol. 2012;61(3):539-42.

34. Rota-Stabelli O, Yang Z, Telford MJ. MtZoa: a general mitochondrial amino acid substitutions model for animal evolutionary studies. Mol Phylogenet Evol. 2009;52(1):268-72.

35. Guindon S, Gascuel O. A simple, fast, and accurate algorithm to estimate large phylogenies by maximum likelihood. Syst Biol. 2003;52(5):696-704.

36. Lupi R, de Meo PD, Picardi E, D'Antonio M, Paoletti D, Castrignano T, et al. MitoZoa: a curated mitochondrial genome database of metazoans for comparative genomics studies. Mitochondrion. 2010;10(2):192-9.

37. Boore JL, Brown WM. Complete sequence of the mitochondrial DNA of the annelid worm Lumbricus terrestris. Genetics. 1995;141(1):305-19.

38. Okimoto R, Chamberlin HM, Macfarlane JL, Wolstenholme DR. Repeated sequence sets in mitochondrial DNA molecules of root knot nematodes (Meloidogyne): nucleotide sequences, genome location and potential for host-race identification. Nucleic Acids Res. 1991;19(7):1619-26.

39. Monnerot M, Solignac M, Wolstenholme DR. Discrepancy in divergence of the mitochondrial and nuclear genomes of Drosophila teissieri and Drosophila yakuba. J Mol Evol. 1990;30(6):500-8.

40. Wolstenholme DR. Animal mitochondrial DNA: structure and evolution. Int Rev Cytol. 1992;141:173-216.

41. Foronda P, Casanova JC, Valladares B, Martinez E, Feliu C. Molecular systematics of several cyclophyllid families (Cestoda) based on the analysis of 18S ribosomal DNA gene sequences. Parasitol Res. 2004;93(4):279-82.
42. Yamasaki H, Ohmae H, Kuramochi T. Complete mitochondrial genomes of Diplogonoporus balaenopterae and Diplogonoporus grandis (Cestoda: Diphyllobothriidae) and clarification of their taxonomic relationships. Parasitol Int. 2012;61(2):260-6.

43. Nakao M, Yokoyama N, Sako Y, Fukunaga M, Ito A. The complete mitochondrial DNA sequence of the cestode Echinococcus multilocularis (Cyclophyllidea: Taeniidae). Mitochondrion. 2002;1(6):497-509.

\section{Submit your next manuscript to BioMed Central and take full advantage of:}

- Convenient online submission

- Thorough peer review

- No space constraints or color figure charges

- Immediate publication on acceptance

- Inclusion in PubMed, CAS, Scopus and Google Scholar

- Research which is freely available for redistribution

Submit your manuscript at www.biomedcentral.com/submit 